\title{
Strong Mode Localization in Nearly Periodic Disordered Structures
}

\author{
Christophe Pierre* and Philip D. Cha $\dagger$ \\ University of Michigan, Ann Arbor, Michigan
}

\begin{abstract}
An investigation of the effects of disorder on the dynamics of nearly periodic structures is presented. Emphasis is placed on the study of mode localization and vibration-confinement phenomena for mistuned assemblies of coupled, multi-degree-of-freedom component systems. Perturbation methods are developed and applied to predict the occurrence of localized modes and analyze their characteristics. Strong localization is shown to occur for weak coupling between component systems. Furthermore, a "modal" coupling parameter is defined that governs the possibility for localization in a given mode. Generally speaking, higher modes are shown to be more susceptible to localization than lower ones, and localization is unavoidable if the mode number is large enough. The occurrence of localization is also shown to be dependent upon the location of the coupling constraint between the component systems.
\end{abstract}

\section{Introduction}

$\mathbf{S}^{1}$ TRUCTURAL systems are commonly investigated by ssuming that their parameters are known precisely, even though manufacturing and material tolerances result in parameter uncertainties and structural irregularities that can affect their dynamics significantly. The assumption of structural regularity is perhaps most critical when studying nominally periodic structures, since it has been observed that the presence of irregularities in such structures may inhibit the propagation of vibration. Depending on the magnitudes of both the disorder and the internal coupling, the irregularities may localize the vibration modes and confine the energy to a region close to the source. This phenomenon, referred to as normal mode localization, has excited considerable interest in solid-state physics ${ }^{1-4}$ over the years and more recently was rediscovered in the field of structural dynamics. ${ }^{5-15}$ A survey of mode localization phenomena in structures can be found in the review paper by Ibrahim. ${ }^{16}$

Nearly periodic structures made of weakly coupled component systems have closely spaced eigenvalues that make them highly sensitive to small irregularities. The modes of such tuned, or periodic, structures extend throughout the structure, whereas the modes of the corresponding mistuned, or disordered, structures undergo drastic changes to become localized about a few component systems. Research by the present author and others has shown that strong mode localization occurs for assemblies of weakly coupled component systems such as chains of coupled pendulums, ${ }^{5-7}$ bladed-disk assemblies, ${ }^{8,9}$ and some large space structures. ${ }^{10,11}$ Disordered multispan structures with irregularly spaced constraints have been shown to be susceptible to mode localization as well, through both theoretical and experimental studies. ${ }^{12,13}$ Most of the previous work on localized vibrations of assemblies of component systems concerns single-degree-of-freedom (DOF) substructures, although the research by Valero and Bendiksen ${ }^{8}$ and Cornwell and Bendiksen ${ }^{11}$ on the occurrence of localization in mistuned shrouded blade assemblies and in large space reflectors, respec-

Received June 8, 1987; revision received Feb. 24, 1988. Copyright (C) American Institute of Aeronautics and Astronautics, Inc., 1988. All rights reserved.

*Assistant Professor, Mechanical Engineering and Applied Mechanics. Member AIAA.

$\dagger$ Graduate Student. tively, should be noted. Both of these studies, however, used a finite-element representation of the subsystems, whereas the present work chooses a component mode synthesis procedure that leads to general results.

The modes of chains of single-DOF oscillators have been extensively studied, ${ }^{5-7}$ and localization has been shown to occur when both the irregularities and the interoscillator coupling are small. In other words, localization will occur if the tuned eigenvalues are clustered in a small band. It was also shown that the degree of localization depends only upon the ratio of disorder to coupling and that localization becomes more pronounced as this ratio increases. In Ref. 7, perturbation methods were used systematically to conduct the investigation. Hodges $^{5,12}$ showed that irregularities may also result in the confinement of forced vibrations near the source of excitation.

Localized modes in disordered structural systems may result in either beneficial or damaging consequences. For instance, localized vibrations in mistuned blade assemblies may be damaging because the stresses remain localized and thus lead to blade fatigue. Serious negative consequences also can be expected for the robustness and stability of active control systems for large space structures if their design is based upon erroneous extended modes of the ordered structure. However, for the same large space structures, localization could also be used as a means of confining vibrations to a region close to the source of disturbance. In all cases, because small irregularities lead to such drastic phenomena, it is important to explore the underlying physical mechanisms of mode localization.

This paper investigates the strong localization of the modes of free vibration of disordered structures made of coupled multi-DOF component systems. In the first part of the paper, the equations of motion are derived by describing each subsystem by its modal representation, and perturbation methods are applied to predict the occurrence of strongly localized modes and analyze their characteristics. These methods, in addition to their low cost, provide an important physical insight into localization. A "modal" coupling is then defined that determines the possibility of strong localization in a given mode. In the second part of the paper, the general theory is applied to a system made of coupled component beams. The motivation for this study is that a cyclic assembly can be regarded as a simple model of a bladed disk. Numerical results are presented, and the localization of the modes is discussed in terms of the strength of the interbeam coupling, the mode number, and the location of the coupling constraint. 


\section{Disordered Assemblies of Component Systems}

\section{Equations of Motion}

The chain of oscillators of Ref. 7 can be generalized by replacing each pendulum with a multi-DOF subsystem. In this part, the modes of vibration of a disordered assembly of $N$ coupled, continuous (or possibly discrete) component systems are studied. Each structural component system is described by its modal representation; hence, the approach is general. The equations of free motion are derived by a component mode analysis ${ }^{17}$ as follows.

Assemblies such as the one shown in Fig. 1 are studied. For the sake of simplicity, one-dimensional component systems are considered, although this can be generalized (for example, see Ref. 6). The ordered, or tuned, assembly consists of identical subsystems. The free modes of the nominal (unperturbed) component system are $\left[\lambda_{i}, \phi_{i}(x)\right]_{i=1, \ldots, M}$, where $M$ is the number of component modes included in the analysis, $\lambda_{i}$ the square of the natural frequency, and $\phi_{i}$ the associated mode shape. When small disorder is introduced, the modes of the $j$ th (decoupled) component system become

$$
\begin{gathered}
\lambda_{i}^{j}=\lambda_{i}\left(1+d \lambda_{i}^{\prime}\right), \quad i=1, \ldots, M, \quad j=1, \ldots, N \\
\phi_{i}^{j}(x)=\phi_{i}(x)+d \phi_{i}^{j}(x), \quad i=1, \ldots, M, \quad j=1, \ldots, N
\end{gathered}
$$

where the subscript $i$ refers to the component mode number and the superscript $j$ designates the component system number; $x$ is the position along the component systems; $d \lambda_{i}^{j}$ and $d \phi_{i}^{j}$ are the $i$ th mode perturbations for the $j$ th component system, resulting from structural and material irregularities. (For simplicity, the domain of the component systems is assumed to remain unperturbed.) Note that $d \lambda_{i}^{j}$ is the dimensionless mistuning, a first-order quantity. These component mode perturbations can be easily determined from the structural parameter perturbations by applying the perturbation theory for the eigenvalue problem; ${ }^{18,19}$ clearly they include first- as well as higher-order terms in the parameter perturbations.

The coupling between the component systems is represented by identical springs (or massless axial members) of stiffness $k$. Small variations of this stiffness throughout the assembly are believed to have small effects (leading to weak localization behavior ${ }^{15}$ ), and thus are not considered. For the $j$ th component system, the coupling constraint is located at $x_{j}$, and the corresponding deflection $w^{j}\left(x_{j}\right)$ is denoted by $w^{j}$.

The deflection of the $j$ th component system is expanded as

$$
w^{j}(x, t)=\sum_{i=1}^{M} \phi_{i}^{j}(x) \eta_{i}^{\prime}(t), \quad j=1, \ldots, N
$$

where $\eta_{i}^{j}$ is the $i$ th normal coordinate for the $j$ th component system. The kinetic energy of the system is

$$
T=\frac{1}{2} \sum_{j=1}^{N} \sum_{i=1}^{M} M_{i}\left(\dot{\eta}_{i}^{j}\right)^{2}
$$

where $M_{i}$ denotes the $i$ th generalized mass for an individual component system. Note that the component modes have not been normalized. However, for a given component mode number, the generalized masses have been chosen to be identical for all component systems. This is natural if perturbation methods for the eigenvalue problem are used to obtain the modes of the mistuned component systems from the ones of the nominal component system, since these methods conserve the generalized masses. The strain energy of the system is

$$
U=\frac{1}{2} \sum_{j=0}^{N} k\left(w^{j}-w^{j+1}\right)^{2}+\frac{1}{2} \sum_{j=1}^{N} \sum_{i=1}^{M} M_{i} \lambda_{i}^{j}\left(\eta_{i}^{j}\right)^{2}
$$

where $M_{i} \lambda_{i}^{j}$ is the $i$ th modal stiffness for the $j$ th component system. By definition, $w^{0}=w^{N+1}=0$. The $N$ constraints are

$$
f^{j}=w^{j}-\sum_{i=1}^{M} \phi_{i}^{j}\left(x_{j}\right) \eta_{i}^{j}(t)=0, \quad j=1, \ldots, N
$$

and the Lagrangian of the system is given by

$$
L=T-U+\sum_{j=1}^{N} \alpha^{j} f^{j}
$$

where the various $\alpha^{j}$ are the Lagrange multipliers. Applying Hamilton's principle, the equations of free motion are found to be, in addition to the constraints in Eq. (6),

$$
\begin{gathered}
M_{i} \ddot{\eta}_{i}^{j}+M_{i} \lambda_{i}^{j} \eta_{i}^{j}+\alpha^{j} \phi_{i}^{j}\left(x_{j}\right)=0, \quad i=1, \ldots, M, \quad j=1, \ldots, N \\
k\left(-w^{j-1}+2 w^{j}-w^{j+1}\right)-\alpha^{j}=0, \quad j=1, \ldots, N
\end{gathered}
$$

Eliminating the Lagrange multipliers and assuming simple harmonic motion of frequency $\omega$ yields

$$
\begin{gathered}
M_{i}\left(-\omega^{2}+\lambda_{i}\right) \bar{\eta}_{i}^{j}+k \phi_{i}^{j}\left(x_{j}\right) \sum_{l=1}^{M}\left[-\bar{\eta}_{l}^{j-1} \phi_{l}^{j-1}\left(x_{j-1}\right)\right. \\
\left.+2 \bar{\eta}_{l}^{j} \phi^{j}\left(x_{j}\right)-\bar{\eta}_{l}^{j+1} \phi_{l}^{j+1}\left(x_{j+1}\right)\right]=0 \\
i=1, \ldots, M, \quad j=1, \ldots, N
\end{gathered}
$$

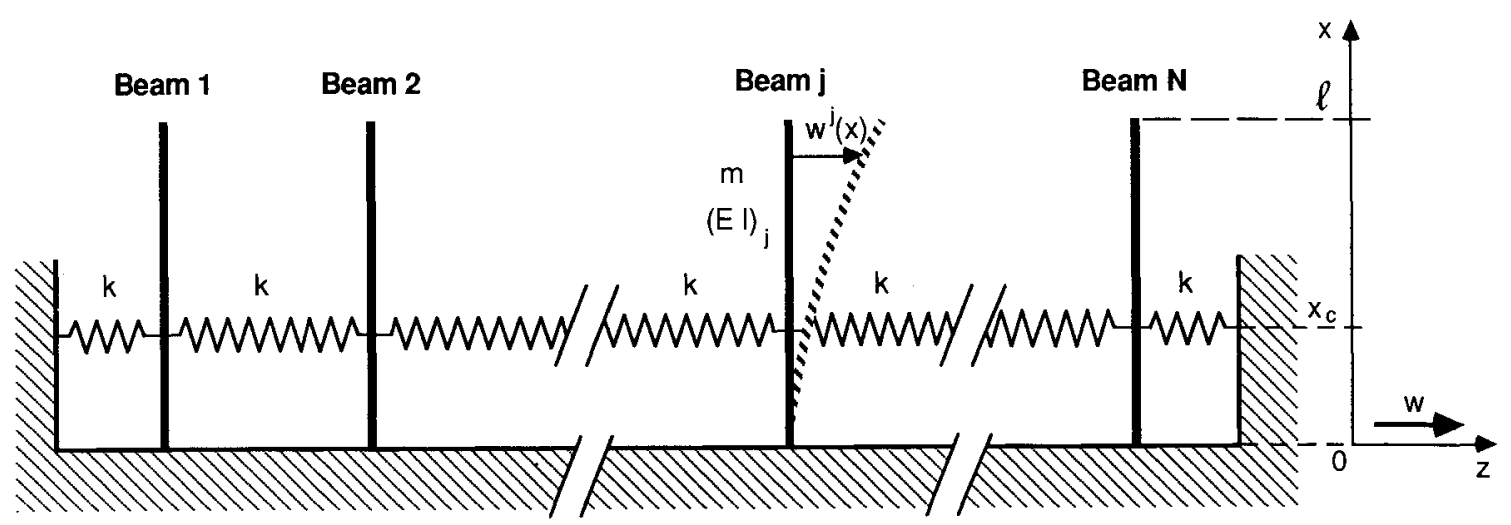

Fig. 1 Assembly of coupled component systems (beams). 
Defining the vector of normal coordinate amplitudes $\bar{\eta}$ of dimension $N M$ as

$$
\bar{\eta}=\left[\bar{\eta}_{1}^{1}, \ldots, \bar{\eta}_{i}^{1}, \ldots, \bar{\eta}_{M}^{1}|\ldots \ldots| \bar{\eta}_{1}^{N}, \ldots, \bar{\eta}_{i}^{N}, \ldots, \bar{\eta}_{M}^{N}\right]^{T}
$$

Equation (10) can be written in matrix form as

$$
\left\{[A]-\omega^{2}[I]\right\} \bar{\eta}=\mathbf{0}
$$

where $[I]$ is the identity matrix and $[A]$ is an $N M$ by $N M$ block tridiagonal matrix given by

$$
[A]=\left[\begin{array}{ccccc}
\ddots & \ddots & {[0]} & \ldots & {[0]} \\
\ddots & \ddots & \ddots & {[0]} & \vdots \\
{[0]} & {\left[a^{j, j-1}\right]} & {\left[a^{j, j}\right]} & {\left[a^{j, j+1}\right]} & {[0]} \\
\vdots & {[0]} & \ddots & \ddots & \ddots \\
{[0]} & \cdots & {[0]} & \ddots & \ddots
\end{array}\right]
$$

The $M$ by $M$ submatrices $\left[a^{k, l}\right]$ are defined by

$$
\left\{\begin{array}{l}
{\left[a^{j, j-1}\right]=-\left[\operatorname{diag}\left(k / M_{i}\right)\right] \phi_{j} \phi_{j-1}^{T}, \quad j=2, \ldots, N} \\
{\left[a^{j, j}\right]=\left[\operatorname{diag}\left(\lambda_{i}^{j}\right)\right]+2\left[\operatorname{diag}\left(k / M_{i}\right)\right] \phi_{j} \phi_{j}^{T}, \quad j=1, \ldots, N} \\
{\left[a^{j, j+1}\right]=-\left[\operatorname{diag}\left(k / M_{i}\right)\right] \phi_{j} \phi_{j+1}^{T}, j=1, \ldots, N-1}
\end{array}\right.
$$

where $\phi_{j}$ is the $M$ vector of modal deflections at $x_{j}$ :

$$
\phi_{j}=\left[\phi_{1}^{\prime}\left(x_{j}\right), \ldots, \phi_{i}^{\prime}\left(x_{j}\right), \ldots, \phi_{M}^{i}\left(x_{j}\right)\right]^{T}
$$

Note that $\phi_{k} \phi_{l}^{T}$ is an $M$ by $M$ matrix that can be regarded as a matrix of modal influence coefficients between the constraint locations of two component systems; $k / M_{i}$ is the coupling natural frequency for the $i$ th component mode. The submatrices constituting $[A]$ correspond to component systems, while the $M$ rows and columns of these submatrices refer to component modes. For an assembly of single-DOF systems, $M=1$ and the submatrices reduce to scalar quantities.

\section{Perturhation Methods}

The occurrence (or nonoccurrence) of strongly localized modes can be predicted by applying perturbation theory for the eigenvalue problem. Moreover, the characteristics of localized modes can be analyzed by a modified perturbation scheme. This has been presented in Ref. 7 for a chain of single-DOF oscillators and is generalized here to an assembly of component systems. In order to study the relative orders of magnitude of the elements of $[A]$, it is convenient to nondimensionalize it by dividing the $i$ th rows of the submatrices $\left[a^{k, h}\right]$ by the corresponding eigenvalue of the nominal component system $\lambda_{i}$. This yields the generalized eigenvalue problem:

$$
\left\{[\widetilde{A}]-\omega^{2}\left[\mathrm{DIAG}\left[\operatorname{diag}\left(1 / \lambda_{i}\right)\right]\right]\right\} \boldsymbol{\eta}=\mathbf{0}
$$

where [DIAG[ $\left.\left.\operatorname{diag}\left(1 / \lambda_{i}\right)\right]\right]$ is an $N M$ by $N M$ diagonal matrix made of $N$ identical $M$ by $M$ diagonal submatrices of generic element $1 / \lambda_{i} ;[\tilde{A}]$ is given by

$$
[\tilde{A}]=\left[\begin{array}{ccccc}
\ddots & \ddots & {[0]} & \ldots & {[0]} \\
\ddots & \ddots & \ddots & {[0]} & \vdots \\
{[0]} & {\left[\tilde{a}^{j, j-1}\right]} & {\left[\tilde{a}^{j, j}\right]} & {\left[\tilde{a}^{j, j+1}\right]} & {[0]} \\
\vdots & {[0]} & \ddots & \ddots & \ddots \\
{[0]} & \cdots & {[0]} & \ddots & \ddots
\end{array}\right]
$$

where

$$
\left\{\begin{array}{l}
{\left[\tilde{a}^{j, j-1}\right]=-\left[\operatorname{diag}\left(R_{i}^{2}\right)\right] \phi_{j} \phi_{j-1}^{T}} \\
{\left[\tilde{a}^{j, j}\right]=\left[\operatorname{diag}\left(1+d \lambda_{i}^{j}\right)\right]+2\left[\operatorname{diag}\left(R_{i}^{2}\right)\right] \phi_{j} \phi_{j}^{T}} \\
{\left[\tilde{a}^{j, j+1}\right]=-\left[\operatorname{diag}\left(R_{i}^{2}\right)\right] \phi_{j} \phi_{j+1}^{T}}
\end{array}\right.
$$

where $R_{i}^{2}=k /\left(M_{i} \lambda_{i}\right)$ is the dimensionless ratio of the spring stiffness to the modal stiffness for the $i$ th component mode or, equivalently, the ratio of the square of the modal coupling natural frequency $k / M_{i}$ to the square of the $i$ th component frequency $\lambda_{i}$; hence, $R_{i}^{2}$ can be regarded as a modal stiffness ratio governing the coupling between component systems. Note that $R_{i}^{2}$ decreases as the component mode number increases, because $\lambda_{i}$ increases with $i$.

For simplicity, identical constraint locations are considered in the subsequent developments, i.e., $x_{c} \equiv x_{j} ; j=1, \ldots, N$. Several cases are discussed.

\section{Strong Coupling in All Component Modes}

Here finite or large values of the stiffness $k$ are considered. It is also assumed that all of the modal stiffness ratios $\left(R_{i}^{2}\right)_{i=1, \ldots . M}$ are finite, i.e., on the order of one. Furthermore, it is assumed that the modal deflections at the constraint location are not small. In brief, the elements of the submatrices $\left[\operatorname{diag}\left(R_{i}^{2}\right)\right] \phi_{k} \phi_{l}^{T}$ are not small, but on the order of one or larger. Then all of the elements of $[\tilde{A}]$ have approximately the same order of magnitude, and the usual perturbation theory for the eigenvalue problem (referred to here as the classical perturbation method) can be used. The unperturbed system is the tuned one, and the perturbation consists of the (modal) mistuning among the component systems. The unperturbed matrix is

$$
\left[\tilde{A}_{0}^{(c)}\right]=\left[\begin{array}{ccccc}
\ddots & \ddots & {[0]} & \ldots & {[0]} \\
\ddots & \ddots & \ddots & {[0]} & \vdots \\
{[0]} & -\left[\tilde{a}_{0}\right] & {[I]+2\left[\tilde{a}_{0}\right]} & -\left[\tilde{a}_{0}\right] & {[0]} \\
\vdots & {[0]} & \ddots & \ddots & \ddots \\
{[0]} & \ldots & {[0]} & \ddots & \ddots
\end{array}\right]
$$

where

$$
\left[\tilde{a}_{0}\right]=\left[\operatorname{diag}\left(R_{i}^{2}\right)\right] \phi \phi^{T}
$$

and $\phi=\left[\phi_{1}\left(x_{c}\right), \ldots, \phi_{M}\left(x_{c}\right)\right]^{T}$ is the vector of modal deflections at the constraint for the nominal component system. The first-order perturbation matrix is

$$
\left[\tilde{\delta A}^{(c)}\right]=\left[\begin{array}{ccccc}
\ddots & \ddots & {[0]} & \ldots & {[0]} \\
\ddots & \ddots & \ddots & {[0]} & \vdots \\
{[0]} & {\left[\widetilde{\delta a}^{j, j-1}\right]} & {\left[\tilde{\delta a}^{j, j}\right]} & {\left[\tilde{\delta a}^{j, j+1}\right]} & {[0]} \\
\vdots & {[0]} & \ddots & \ddots & \ddots \\
{[0]} & \cdots & {[0]} & \ddots & \ddots
\end{array}\right]
$$

where

$$
\left\{\begin{array}{l}
{\left[\widetilde{\delta a}^{j, j-1}\right]=-\left[\operatorname{diag}\left(R_{i}^{2}\right)\right]\left[\delta \phi^{j, j-1}\right]} \\
{\left[\widetilde{\delta a}^{j, j}\right]=\left[\operatorname{diag}\left(d \lambda_{i}^{j}\right)\right]+2\left[\operatorname{diag}\left(R_{i}^{2}\right)\right]\left[\delta \phi^{j, j}\right]} \\
\left.\left[\widetilde{\delta a}^{j, j+1}\right]=-\operatorname{diag}\left(R_{i}^{2}\right)\right]\left[\delta \phi^{j, j+1}\right]
\end{array}\right.
$$

and $\left[\delta \phi^{k, l}\right]$ is given to the first order by

$\left[\delta \phi^{k, l}\right]=\phi\left(d \phi^{\zeta}\right)^{T}+d \phi^{k} \phi^{T}$ where $d \phi^{k}=\left[\ldots, d \phi_{i}^{k}\left(x_{c}\right), \ldots\right]^{T}$

The second-order perturbation matrix also could be easily obtained. The modes of the mistuned assembly are perturbations of the ones of the tuned system and are certainly not strongly localized. Note that this perturbation approach only requires 
the solution of the tuned system, which can be obtained by taking advantage of its periodicity properties.

\section{Weak Coupling in All Component Modes}

Here the stiffness $k$ and the modal stiffness ratios $\left(R_{i}^{2}\right)_{i=1, \ldots, M}$ are assumed to be small, on the order of or smaller than the dimensionless mistunings $d \lambda_{i}^{j}$ :

$$
R_{i}^{2}=\frac{k}{M_{i} \lambda_{i}} \ll 1, \quad R_{i}^{2} \leq O\left(d \lambda_{i}^{\prime}\right), \quad i=1, \ldots, M
$$

Note that if the condition of Eq. (19) is met for $i=1$, it is likely to be verified for $i>1$ as well, since $R_{i}^{2}$ decreases as $i$ increases.

For small $k$ the natural frequencies of the tuned assembly are clustered in $M$ groups, or bands, with $N$ frequencies in each narrow band. The width of these clusters decreases with $k$, and the introduction of mistuning makes the frequencies constituting the bands move apart slightly. The classical perturbation approach fails to analyze this small coupling case, because the mode shape perturbations become large when disorder is introduced (due to the closeness of the tuned eigenvalues), which violates the underlying assumptions of asymptotic expansions. ${ }^{6,10,14}$ This can be readily observed from the perturbation expression, Eq. (A5), in Appendix A. Indeed, this failure of the perturbation method can be exploited as a means of predicting the occurrence of strong localization (see Ref. 14).

Therefore, the perturbation scheme has to be modified as follows for the small coupling case. Since in Eq. (16) all of the elements of $[\tilde{A}]$ but the diagonal ones are small, the coupling between component systems, as well as the mistuning, ought to be treated as a perturbation. However, if both mistuning and coupling are considered perturbations, the unperturbed matrix becomes the identity matrix, leading to $M N$-fold multiple eigenvalues for the unperturbed system $\lambda_{i}$, corresponding to the modes of identical decoupled component systems. Even though the perturbation theory could still be applied to these multiple eigenvalues, physical insight into mode localization would be lost. Therefore, the key idea is to introduce mistuning in the unperturbed system ${ }^{7}$ in order to split the unperturbed eigenvalues. This results in the (modified) unperturbed matrix

$$
\left[\tilde{A}_{0}^{(m)}\right]=\left[\operatorname{DIAG}\left[\operatorname{diag}\left(1+d \lambda_{i}^{j}\right)\right]\right]
$$

and in the (modified) perturbation matrix

$$
\left[\widetilde{d A}^{(m)}\right]=\left[\begin{array}{ccccc}
\ddots & \ddots & {[0]} & \ldots & {[0]} \\
\ddots & \ddots & \ddots & {[0]} & \vdots \\
{[0]} & -\left[\widetilde{d a}^{j, j-1}\right] & 2\left[\widetilde{d a}^{j, j}\right] & -\left[\widetilde{d a}^{j, j+1}\right] & {[0]} \\
\ddots & {[0]} & \ddots & \ddots & \ddots \\
{[0]} & \cdots & {[0]} & \ddots & \ddots
\end{array}\right]
$$

where

$$
\left[\widetilde{d a}^{k, l}\right]=\left[\operatorname{diag}\left(R_{i}^{2}\right)\right] \phi_{k} \phi_{l}^{T}
$$

The elements of $\left[\tilde{A}_{0}^{(m)}\right]$ and $\left[\widetilde{d A}^{(m)}\right]$ are on the order of one and of the first order, respectively. Since mistuning among subsystems is usually random, the unperturbed eigenvalues $\lambda_{i}(1+$ $d \lambda_{i}^{j}$ ) are likely to be simple. Also, note that Eqs. (20) and (21) are a straightforward generalization of the method developed for a chain of coupled pendulums. ${ }^{7}$

The unperturbed solution consists of the modes of the decoupled mistuned component systems, which have slightly different natural frequencies. It is given by

$$
\begin{aligned}
& \left\{\begin{array}{l}
\lambda_{o p}^{(m)}=\lambda_{i}\left(1+d \lambda_{i}^{j}\right) \\
\overline{\boldsymbol{\eta}}_{o p}^{(m)}=\boldsymbol{e}_{p}
\end{array}\right. \\
& p=(j-1) M+i, \quad j=1, \ldots, N, \quad i=1, \ldots, M
\end{aligned}
$$

where $e_{p}$ is the $p$ th canonical vector in an $N M$ dimensional space, consisting of zeros except for a one at the $p$ th location. Note that the $p$ th (modified) unperturbed mode is the $j$ th mode of the $i$ th frequency band.

The perturbation matrix introduces (small) coupling between the component systems. Since the perturbed modes are perturbations of decoupled oscillations at different natural frequencies, they are expected to be strongly localized about individual component systems. The modes corresponding to the $j$ th component system consist primarily of oscillations of the $j$ th system and of oscillations of lesser magnitude of its nearest neighbors, the propagation range depending on the modal coupling and mistuning. It is remarkable that the modified perturbation method predicts localized modes without requiring one to solve the eigenvalue problem. The perturbation theory for the eigenvalue problem (see Appendix A and Refs. 18 and 19) can be readily applied to Eqs. (20-22). The first-order (modified) eigenvalue perturbation is

$$
\delta \lambda_{p}^{(m)}=\frac{\left(\overline{\boldsymbol{\eta}}_{o p}^{(m)}\right)^{T}\left[\widetilde{d A}^{(m)}\right] \overline{\boldsymbol{\eta}}_{o p}^{(m)}}{1 / \lambda_{i}}=2 R_{i}^{2}\left[\phi_{i}^{j}\left(x_{c}\right)\right]^{2} \lambda_{i}
$$

Hence, the first-order perturbed eigenvalues are given by

$$
\lambda_{p}^{(m)}=\lambda_{i}\left\{1+d \lambda_{i}^{j}+2 R_{i}^{2}\left[\phi_{i}^{j}\left(x_{c}\right)\right]^{2}\right\}
$$

The effects of (small) coupling and mistuning on the system eigenvalues are now clear. Note that the band character of the eigenvalues is evident from Eq. (24). The first-order eigenvector perturbation is

$$
\begin{aligned}
\overline{\boldsymbol{\delta}}_{p}^{(m)}=\sum_{\substack{q=1 \\
q \neq p}}^{N M} \frac{\overline{\boldsymbol{\eta}}_{o q}^{(m)}\left[\widetilde{d A}^{(m)}\right] \overline{\boldsymbol{\eta}}_{o p}^{(m)}}{\left(1 / \lambda_{i^{\prime}}\right)\left(\lambda_{o p}^{(m)}-\lambda_{o q}^{(m)}\right)} \overline{\boldsymbol{\eta}}_{o q}^{(m)}, \\
p=(j-1) M+i, \quad q=\left(j^{\prime}-1\right) M+i^{\prime}
\end{aligned}
$$

The perturbed vector of normal coordinate amplitudes is readily obtained as $\overline{\boldsymbol{\eta}}_{p}=\boldsymbol{e}_{p}+\overline{\boldsymbol{\delta}}_{p}^{(m)}$, from which the perturbed continuous mode shape can be computed using Eq. (3).

In order to obtain physical insight into these results, Eq. (25) can be approximated by using the fact that the modified unperturbed eigenvalues are clustered in narrow bands. One has

$$
\frac{\lambda_{o p}^{(m)}-\lambda_{o q}^{(m)}}{\lambda_{i^{\prime}}}=\frac{\lambda_{i}-\lambda_{i^{\prime}}+\lambda_{i} d \lambda_{i}^{j}-\lambda_{i^{\prime}} d \lambda_{i^{\prime}}^{l^{\prime}}}{\lambda_{i^{\prime}}}
$$

which is small (first order) only if $i=i^{\prime}$, i.e., if both eigenvalues belong to the same group. The other terms in the summation, Eq. (25), yield small (at least one order of magnitude smaller) perturbations and can be neglected. Furthermore, for $i=i^{\prime}$, the only nonzero terms in the summation are for $j^{\prime}=j-1$ and $j+1$, because of the tridiagonality of $\left[\widetilde{d A}^{(m)}\right]$, yielding

$$
\begin{aligned}
& \overline{\boldsymbol{\delta}}_{p}^{(m)}=-\frac{R_{i}^{2} \phi_{i}^{j}\left(x_{c}\right) \phi_{i}^{j-1}\left(x_{c}\right)}{d \lambda_{i}^{j}-d \lambda_{i}^{j-1}} \overline{\boldsymbol{\eta}}_{o p^{\prime}}^{(m)}-\frac{R_{i}^{2} \phi_{i}^{j}\left(x_{c}\right) \phi_{i}^{j+1}\left(x_{c}\right)}{d \lambda_{i}^{j}-d \lambda_{i}^{j+1}} \overline{\boldsymbol{\eta}}_{o p^{\prime \prime}}^{(m)} \\
& \quad+\text { Higher Order Terms (HOT) }
\end{aligned}
$$

where $p^{\prime}=(j-2) M+i$ and $p^{\prime \prime}=j M+i$. For $j=1$ (respectively $j=N$ ), only the second (respectively first) term appears in the right-hand side of Eq. (27). Note that for such a first-order calculation, only the two nearest neighbors of the $j$ th component system are perturbed, leading to a mode shape strongly localized about the $j$ th system. A second-order analysis could also be easily developed and would lead to a perturbation of the four nearest neighbors (see Ref. 7). In general, the order of the perturbation analysis should be determined by the degree of localization of the modes, less strongly localized modes requiring higher-order modified perturbation analyses. 
Equation (27) can also be written as

$$
\overline{\delta \boldsymbol{\eta}}_{p}^{(m)}=\frac{1}{1 / b_{i}^{j-1}-1 / b_{i}^{j}} \overline{\boldsymbol{\eta}}_{o p^{\prime}}^{(m)}+\frac{1}{1 / b_{i}^{j+1}-1 / b_{i}^{j}} \overline{\boldsymbol{\eta}}_{o p^{\prime}}^{(m)}
$$

where $b_{i}^{j}=R_{i}^{2}\left[\phi_{i}\left(x_{i}\right)\right]^{2} / d \hat{\lambda}_{i}^{j}$ is a ratio of modal coupling to disorder [for simplicity it is assumed here that $\phi_{i}^{j}\left(x_{c}\right) \equiv \phi_{i}\left(x_{c}\right)$, independent of $j$ ]. The expression Eq. (28) shows that the degree of localization in the $i$ th group of modes depends only on the ratios $R_{i}^{2}\left[\phi_{i}\left(x_{c}\right)\right]^{2} / d \lambda_{i}^{j}$ of modal coupling to modal disorder-a result similar to the one derived for chains of coupled pendulums. ${ }^{5,7}$ Here, however, it is obtained for an assembly of multiDOF component systems.

\section{General Case}

The coupling is unlikely to be either strong or weak in all component modes. In general, a finite (not small) value of $k$ leads to finite values of $R_{i}^{2}$ for the lower groups of modes and to small values for the higher groups. Then the lower modes remain extended (or only weakly localized), whereas for $i$ greater than some threshold value, strong localization occurs. Moreover, localization is seen to become more and more pronounced as the group number $i$ increases (provided the modal mistunings $d \lambda_{i}^{j}$ are all first-order for various $i$ ), since $R_{i}^{2}$ decreases as $\lambda_{i}$ increases. In the limit $i \rightarrow \infty$, the mistuned modes become decoupled, whereas the tuned ones remain extended. Furthermore, for any $k$ (even arbitrarily large) and for any mistuning (no matter how small), there exists a group number $i^{*}$ such that for $i>i^{*}$, all modes become strongly localized. The conclusion is that the higher groups of modes are always localized, the lower bound for the occurrence of localization depending on $k$ and on the mistuning strength.

Another interesting comment concerns the values of the modal deflections at the constraint location $\phi_{i}^{j}\left(x_{c}\right)$. These deflections determine the magnitude of the coupling terms in $[\tilde{A}]$ (and, therefore, the suitable perturbation schemes) as much as the modal stiffness ratios do. Finite values of $\phi_{i}^{j}\left(x_{c}\right)$ have little effect on the coupling between component systems and thus on mode localization. On the other hand, if the constraint is close to a node of the $i$ th mode, then $\phi_{i}^{j}\left(x_{c}\right)$ is very small (or zero), leading to small coupling between the component systems for the $i$ th group of modes. In this case, even though $R_{i}^{2}$ may be finite or large, the $i$ th rows of the submatrices [diag $\left.\left(R_{i}^{2}\right)\right] \phi_{k} \phi_{l}^{T}$ are small, leading to small effective coupling in the $i$ th group of modes and, therefore, to a localized $i$ th group of modes. Also, the $i$ th group may be localized, whereas the neighboring $(i-1)$ th and $(i+1)$ th groups remain extended. This is achieved if $R_{i+1}^{2}$ is finite and the constraint is located close to one of the nodes of the $i$ th component mode.

Therefore, the occurrence of localization in a group of modes is determined by the relative magnitudes of 1) the modal mistuning, and 2) the modal coupling, defined as the product of the modal stiffness ratio and the modal deflection at the constraint $R_{i}^{2} \phi_{i}^{j}\left(x_{c}\right)$. Strong localization occurs if the latter is of the order of or smaller than the former. In general, a variety of cases can be encountered: 1) all modes strongly localized, 2) extended lower modes and localized higher modes, and 3) extended lower modes except for one group that is localized due to the location of the constraint. It is important to emphasize that if $M$ is chosen large enough, the higher groups of modes always will be localized.

The analysis of both extended and localized modes for a given mistuning configuration can be easily performed by combining the classical and modified perturbation methods. If the coupling for the $i$ th group $R_{i}^{2} \phi_{i}^{j}\left(x_{c}\right)$ is finite, only $d \lambda_{i}^{j}$ must be considered as a perturbation in the $i$ th rows of the submatrices [Eq. (16)]. Conversely, if $R_{i}^{2} \phi_{i}^{j}\left(x_{c}\right)$ is on the order of $d \lambda_{i}^{j}$ or smaller, the coupling terms in the $i$ th rows of the submatrices are considered as perturbations, and $d \lambda_{i}^{j}$ belongs to the unperturbed matrix.

\section{Localization for Coupled Beam Assemblies}

\section{Equations of Motion}

The analysis of Sec. II is applied to the disordered assembly of coupled component beams shown in Fig. 1. Each component system is a uniform cantilevered beam. The square of the natural frequencies of the nominal component beam are $\lambda_{i}=\bar{\omega}_{i}^{2}\left(E I / m l^{4}\right)$, where $\bar{\omega}_{i}$ is the $i$ th dimensionless frequency of a uniform clamped-free beam (the first six values are listed in Appendix B), and $E I, m$, and $l$ are the beam's stiffness, mass per unit length, and length, respectively. It is assumed that mistuning originates from discrepancies in the terms $\left(E I / \mathrm{ml}^{4}\right)$. The eigenvalues of the individual component systems then can be written as

$$
\lambda_{i}^{j}=\bar{\omega}_{i}^{2}\left(\frac{E I}{m l^{4}}\right)_{j}=\bar{\omega}_{i}^{2}\left(\frac{E I}{m l^{4}}\right)\left(1+d \lambda^{j}\right)
$$

where $d \lambda^{j}$ is the dimensionless mistuning for the $j$ th component system. Note that mistuning is the same in all component modes. Nondimensionalizing the eigenvalues by the nominal value $\left(E I / \mathrm{ml}^{4}\right)$ yields

$$
\bar{\lambda}_{i}^{j}=\bar{\lambda}_{i}\left(1+d \lambda^{j}\right)
$$

Since no domain perturbation is considered, all beams have the same length. For simplicity, it is further assumed that mistuning originates from discrepancies in the stiffness $E I$, not in the mass $m$. This results in identical generalized masses for all component systems without introducing a mode shape perturbation $d \phi_{i}^{j}$. Moreover, the generalized masses $M_{i}=m l \equiv M$ are also independent of the mode number. In general, of course, mistuning could originate from local inhomogeneities or boundary condition variations. This would result in different component mode perturbations than the ones given in Eq. (29), but these could be easily calculated by applying the perturbation theory for the continuous eigenvalue problem. ${ }^{19,20}$ Therefore, the mode shapes of the component beams are given by

$$
\begin{aligned}
& \phi_{i}^{j}(x)=\cos \beta_{i} x-\cosh \beta_{i} x+\frac{\sin \beta_{i} l-\sinh \beta_{i} l}{\cos \beta_{i} l+\cosh \beta_{i} l} \\
& \quad \times\left(\sin \beta_{i} x-\sinh \beta_{i} x\right)
\end{aligned}
$$

where $\left(\beta_{i} l\right)^{4}=\bar{\omega}_{i}^{2}$, and $\phi_{i}^{j} \equiv \phi_{i}$ independent of $j$, i.e., there is no component mode shape perturbation due to mistuning.

In the next step, Eqs. (12-14) are readily applied to the beam system. An important simplification is that the modal deflection vector $\phi$ is now the same for all component systems. Furthermore, the equations are made dimensionless by dividing them by the nominal value $E I / \mathrm{ml}^{4}$, resulting in the introduction of dimensionless eigenvalues $\bar{\omega}^{2}$ and of the dimensionless coupling stiffness $\bar{k}=k /\left(E I / l^{3}\right)$. The equations of free motion are

$$
\left\{[\bar{A}]-\bar{\omega}^{2}[I]\right\} \bar{\eta}=0
$$

where

$[\bar{A}]=\left[\begin{array}{ccccc}\ddots & \ddots & {[0]} & \ldots & {[0]} \\ \ddots & \ddots & \ddots & {[0]} & \vdots \\ {[0]} & -\bar{k} \phi \phi^{T}\left[\operatorname{diag}\left(\bar{\lambda}_{i}\left(1+d \lambda^{j}\right)\right)\right]+2 \bar{k} \phi \phi^{T}-\bar{k} \phi \phi^{T} & {[0]} \\ \vdots & {[0]} & \ddots & \ddots & \ddots \\ {[0]} & \cdots & {[0]} & \ddots & \ddots\end{array}\right]$

Equation (32) could also be written in the form of Eqs. (15) and (16) by dividing the $i$ th rows of the submatrices by $\bar{\lambda}_{i}$, resulting in the modal stiffness ratios $R_{i}^{2}=\bar{k} / \bar{\lambda}_{i}$. Once the 

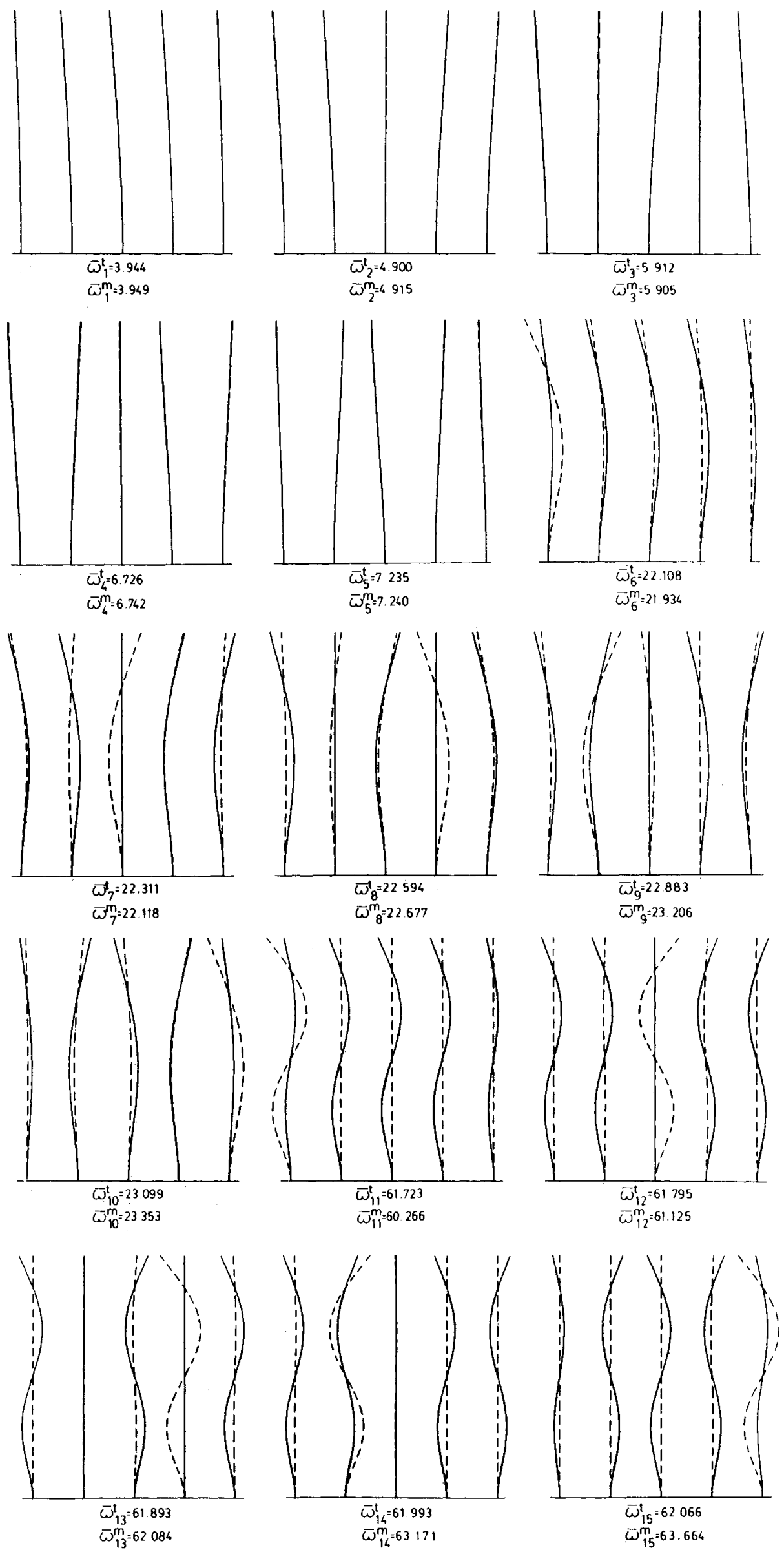

Fig. 2 Modes of tuned $\left(-, \bar{\omega}^{t}\right)$ and mistuned $\left(---, \bar{\omega}^{m}\right)$ assemblies of five beams for $\bar{k}=3, x_{c}=1$, and $M=6$. The mistuning is $d \lambda^{1}=-0.055$, $d \lambda^{2}=0.04, d \lambda^{3}=-0.025, d \lambda^{4}=0.006$, and $d \lambda^{5}=0.055$. 

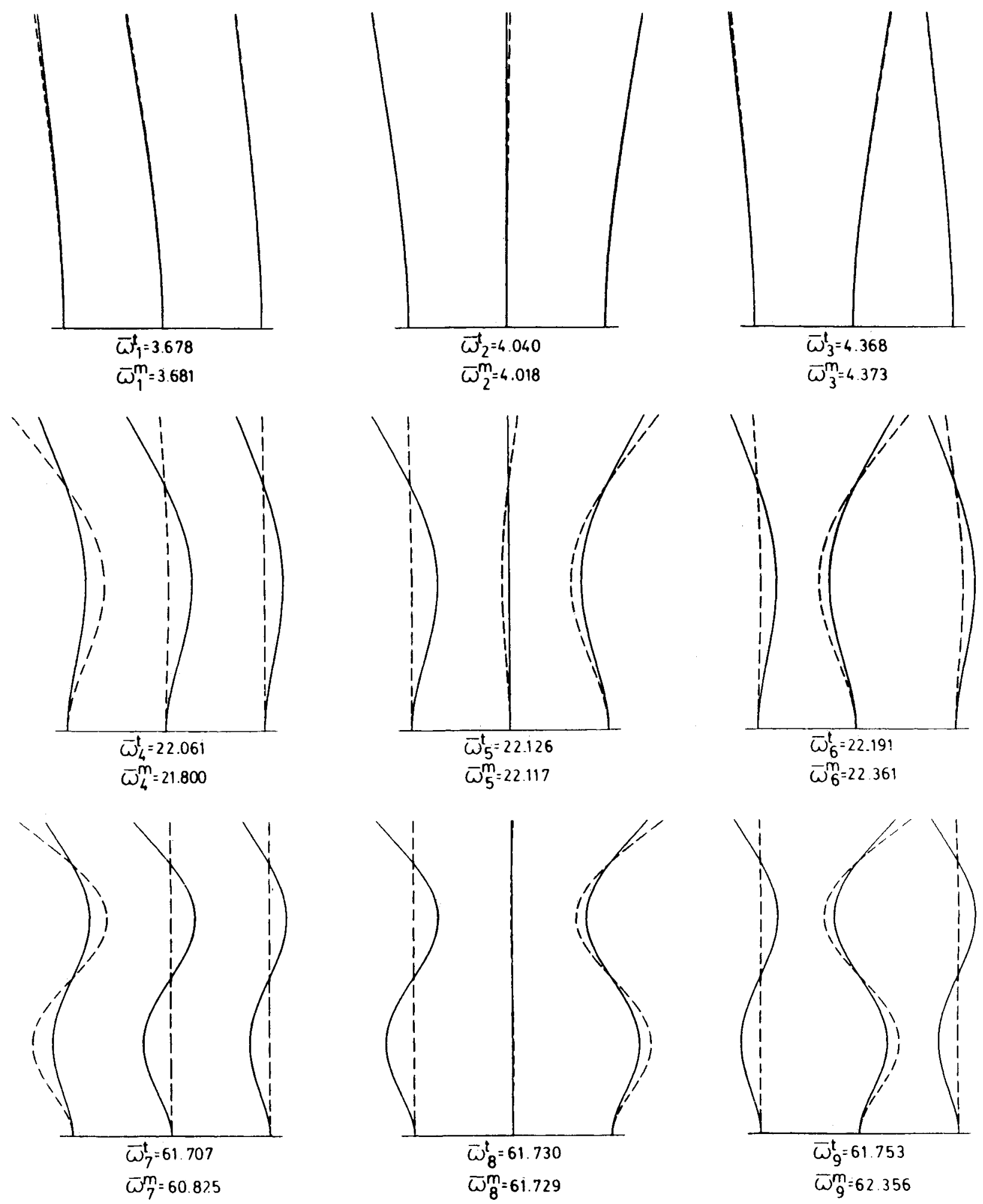

Fig. 3 Modes of tuned $\left(-, \bar{\omega}^{t}\right)$ and mistuned (---, $\left.\bar{\omega}^{m}\right)$ assemblies of three beams for $\bar{k}=0.5, x_{c}=1$, and $M=6$. The mistuning is $d \lambda^{1}=-0.03$, $d \lambda^{2}=0.02$, and $d \lambda^{3}=-0.0$.

eigensolution of $[\bar{A}]$ has been obtained, the continuous mode shapes are computed using Eq. (3).

\section{Results}

Unless otherwise stated, results were obtained by direct solution procedure, not by perturbation methods. Tuned and mistuned assemblies of three and five component beams were studied. The random-like mistuning distributions had small standard deviation estimates of 2.5 and $4.5 \%$ for three and five beams, respectively, and are listed in the figure captions. Only the value of the coupling stiffness $\bar{k}$ was varied for a given small mistuning, because the degree of localization depends only upon the ratio of coupling to mistuning. Therefore, when localization occurs, an increase in coupling is equivalent to a decrease in mistuning. Moreover, several constraint locations were considered.

Figure 2 shows the lower 15 modes of tuned and mistuned assemblies of five beams connected at their free ends for $\bar{k}=3$, i.e., the spring stiffness equals the static stiffness of the nominal beam. The corresponding natural frequencies are also listed. Observe that the high frequencies are clustered in groups of 
five. Even though the lower five frequencies do not have a small band character, the 6th to 10 th and 11 th to 15 th frequencies constitute two narrow clusters. The band width is seen to decrease rapidly as the group number increases, which is a consequence of the corresponding decrease in the modal stiffness ratio $R_{i}^{2}$. The lower five frequencies do not exhibit a small band character because the coupling in the first group of modes is not small.

One observes in Fig. 2 that all of the modes of the tuned system are extended, i.e., all beams vibrate with comparable amplitudes (except when symmetry causes a beam to have a zero deflection). Also, in a given group of modes, a single component mode contributes largely to the motion. For instance, in the lower five modes the beams deflect primarily in the first component mode. In general, in the $i$ th group of modes the beams vibrate primarily in the $i$ th component mode. This can be explained by noting that the interbeam coupling is not strong enough to distort significantly the deflection shapes of the coupled assembly from the decoupled component modes. As will be seen later, for large values of $k$ several component modes may contribute significantly to the modes of the assembly.

For the mistuned modes in Fig. 2, the lower five modes ( first group) are very similar to the corresponding tuned ones and do not become localized. In the second group, the modes undergo significant changes when small mistuning is introduced and become partially localized. In the third band, the mistuned modes are drastically different from the tuned ones, each mis- tuned mode becoming strongly localized about one component beam. Localization is so pronounced that the modes are almost decoupled, i.e., they consist of oscillations of decoupled mistuned beams. The conclusion is that localization becomes more severe as the group number increases. This was predicted in Sec. II by noting that the modal coupling decreases rapidly as the component mode number increases, which causes the offdiagonal coupling terms in Eq. (33) to become small relative to the diagonal terms $\bar{\lambda}_{i}$. For the system of Fig. 2, the modal stiffness ratios are $R_{1}^{2}=0.24, R_{2}^{2}=0.006, R_{3}^{2}=8.10^{-4}$, and $R_{4}^{2}=2.10^{-4}$, explaining that very strong localization occurs for $i \geq 3$. As $i$ continues to increase, stronger and stronger localization occurs. Therefore, for the mistuned system, all the higher modes are strongly localized, and an analysis not accounting for small disorder would yield erroneous results. Here, the confinement effect is, indeed, spectacular, as the mistuning standard deviation is only $4.5 \%$, and the coupling stiffness is not small but equal to the beam's static stiffness.

Since the mistuned modes of the first group remain extended, they are merely perturbations of the tuned modes. Conversely, the mistuned modes of the third and higher groups are perturbations of the modes of the decoupled mistuned system. To illustrate this point, a combined classical and modified perturbation method has been applied to a mistuned three-beam assembly whose first group of modes remains extended while the second group becomes strongly localized. For simplicity, two component modes have been considered in the analysis (good convergence of the component mode procedure was already
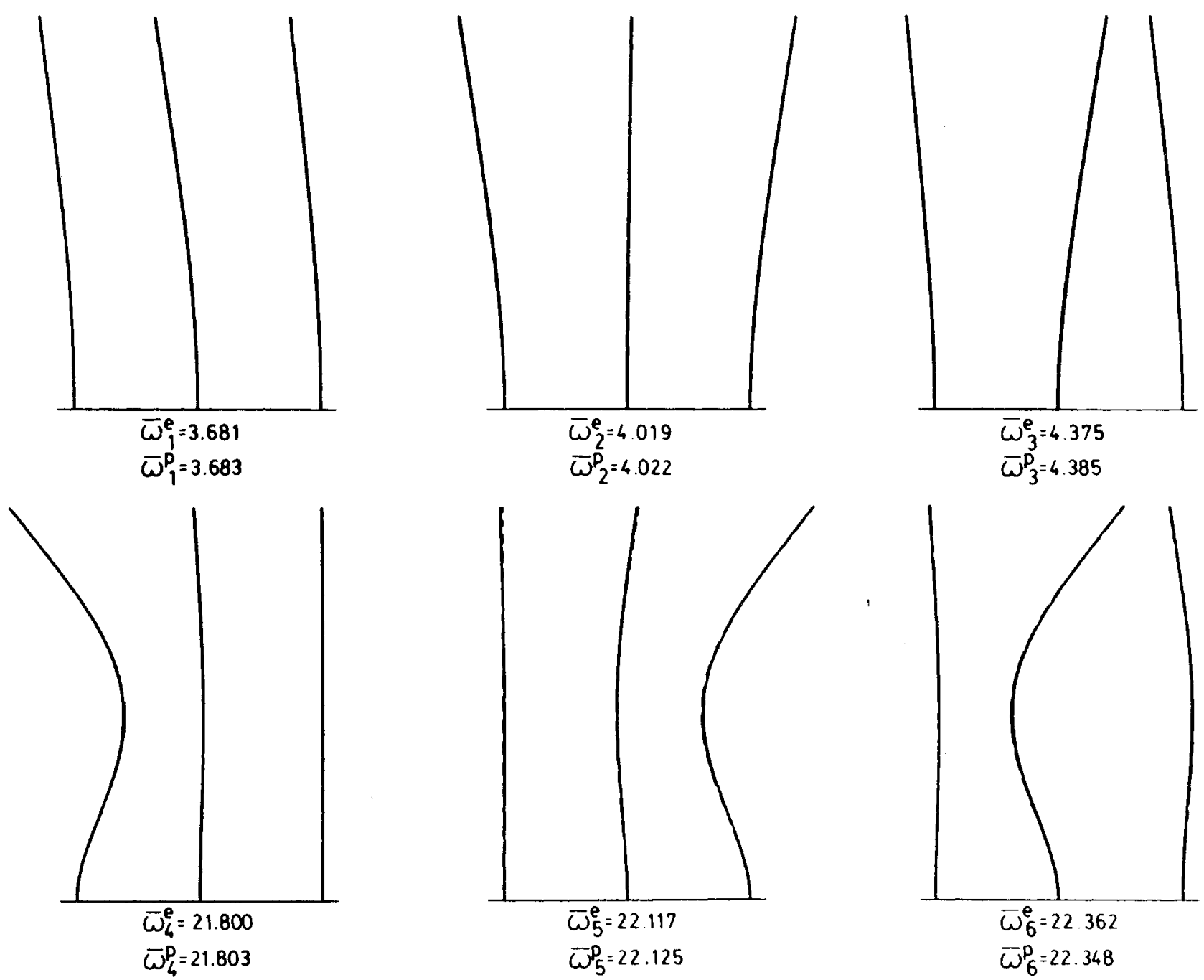

Fig. 4 Comparison of the modes of a mistuned assembly of three beams obtained by the exact method $\left(-, \bar{\omega}^{e}\right)$ and the combined classical and modified perturbation method $\left(--, \bar{\omega}^{p}\right) ; \bar{k}=0.5, x_{c}=1$, and $M=2$. Mistuning is as in Fig. 3 . 

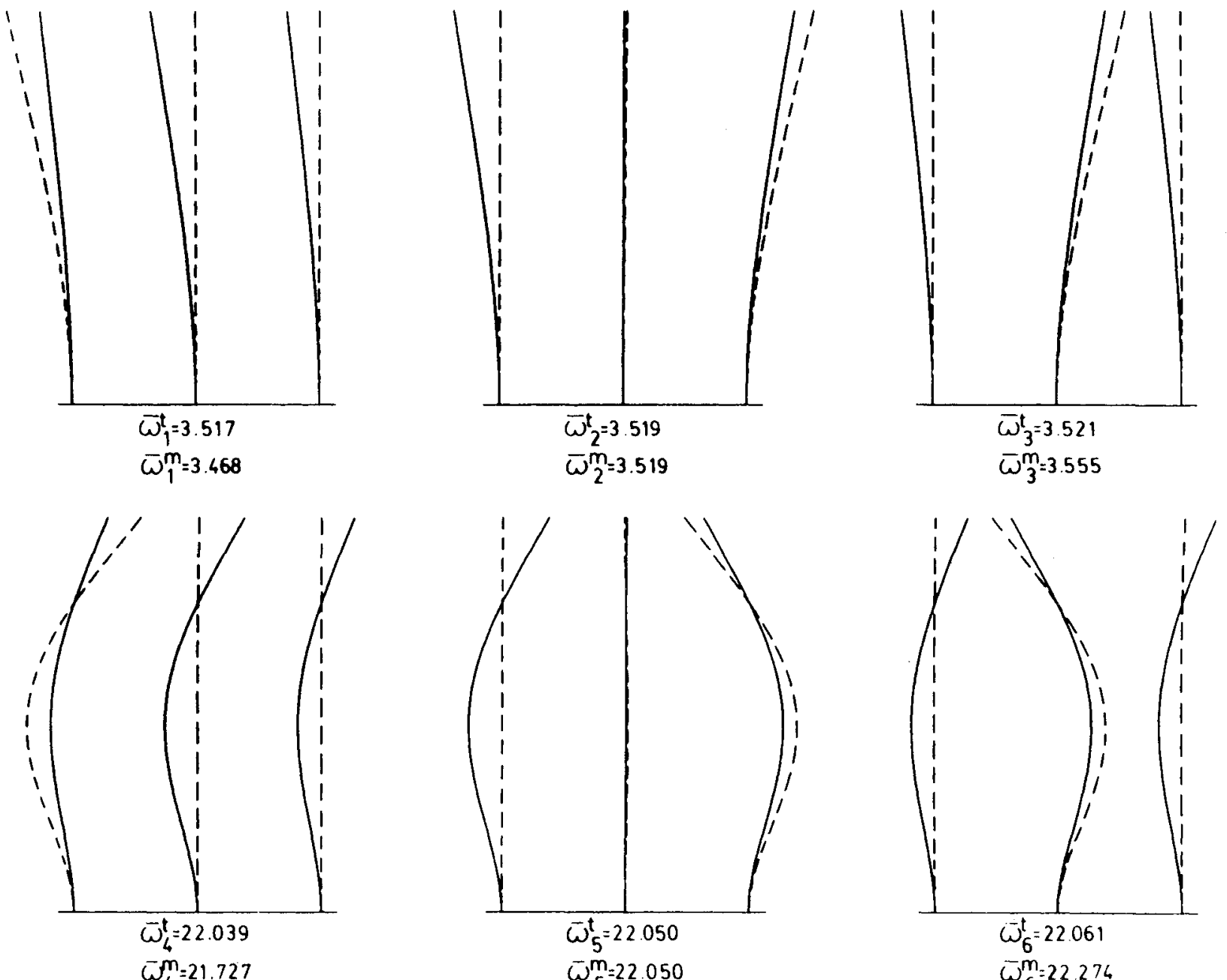

\section{$\bar{\omega}_{5}^{m}=22.050$}
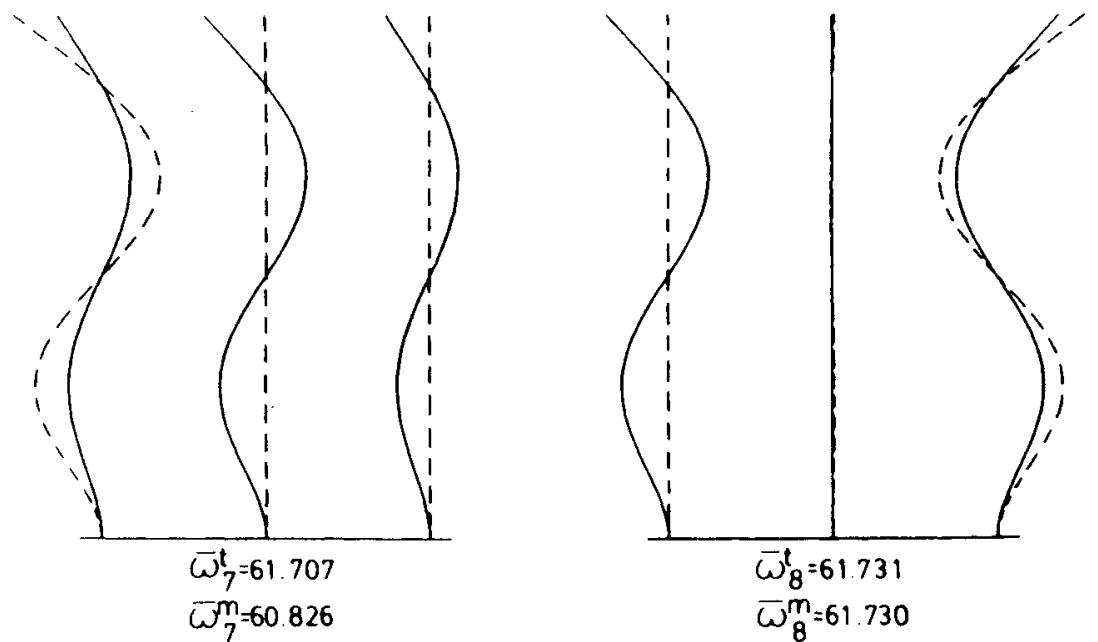

$\bar{\omega}_{6}^{m}=22.274$

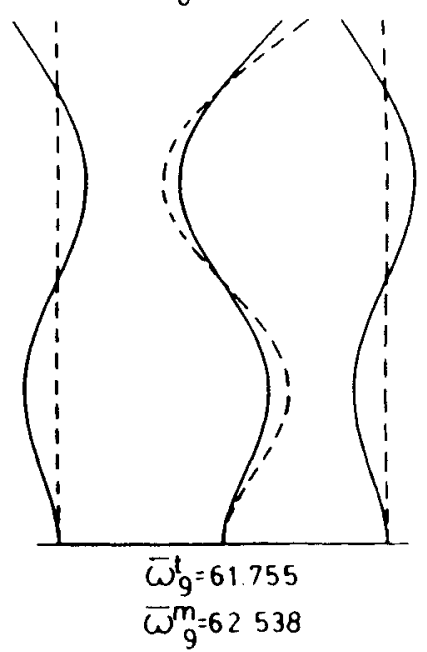

Fig. 5 Modes of tuned $\left(-, \bar{\omega}^{t}\right)$ and mistuned $\left(--, \bar{\omega}^{m}\right)$ assemblies of three beams for $\bar{k}=10, x_{c}=0.1$, and $M=6$. Mistuning is as in Fig. 3 .

achieved in this case for $M=2$ ). The classical scheme defined in Eqs. (17) and (18) has been applied to the first group of modes, whereas the modified method given by Eqs. (20) and (21) has been used for the second group. Therefore, in Eq. (33), only the mistuning $\bar{\lambda}_{1} d \lambda^{j}$ was included in the first rows of the submatrices constituting the perturbation matrix, while all the coupling terms were included in their second rows. The unperturbed modes consist of the lower three modes of the tuned system and of the three second modes of the decoupled mistuned beams. The formulas given in Appendix A for the firstorder eigensolution perturbation were applied. The "exact" modes of the tuned and mistuned systems are displayed in Fig. 3 for $k=0.5$ and $x_{c}=1$. Observe that the first group remains extended, while the higher groups are strongly localized. The mistuned modes obtained by both the exact method and the first-order perturbation method are displayed in Fig. 4 for the first two groups. Excellent agreement is observed. For instance, the maximum error is $0.2 \%$ for the natural frequencies. As predicted, the first group is not localized by mistuning, whereas the second group undergoes strong localization. Of course, higher groups of modes would even be more strongly localized. This example demonstrates that perturbation methods have 
the ability to predict and analyze localization without solving the mistuned eigenvalue problem. Moreover, important physical insight is gained through these methods. Note also that a second-order perturbation analysis could be easily implemented.

Figure 5 displays the tuned and mistuned modes of a threebeam assembly for $\bar{k}=10$ and $x_{c}=0.1$, i.e., the coupling stiffness is large, and the constraint is located near the clamped base of the beams. One observes that all groups of modes exhibit a small-band character and that all of the mistuned modes, even the lower three, become strongly localized. Again, localization is stronger in the higher modes. The occurrence of localization in the first frequency cluster can be explained by noting that, even though $\bar{k}$ is large, the coupling between component systems in the first group of modes is determined by $\vec{k} \phi_{1}\left(x_{c}\right) / \bar{\lambda}_{1}$, which is small because of the small value of $\phi_{1}(0.1)$. Thus, even though the coupling stiffness is 20 times the one of Fig. 3, all modes are strongly localized because the coupling is rendered small by the small modal deflections at the constraint location.

Figure 6 is for beams very strongly coupled at their tips $(k=100)$. There is no small-band character for the first nine frequencies and, accordingly, the lower nine modes do not become localized. However, the 10th to 12 th and 13th to 15 th frequencies are clustered in two groups of small widths. Weak localization occurs in the fourth band, whereas the modes of the fifth group are strongly localized. This can be easily explained by considering Eq. (33), as the modal stiffness ratios $\bar{k} / \bar{\lambda}_{i}$ are very large for the lower modes but become small in the fourth and fifth bands, allowing localization to occur. The conclusion is that even for (arbitrarily) large coupling, localized modes always occur at high frequencies, the threshold value being determined by the value of the spring stiffness and by the location of the constraint.

It is also worth noting that in Fig. 6 the lower six modes are quite different from the ones shown in Figs. 2-5 for smaller values of $\bar{k}$, both in terms of mode shapes and natural frequencies. This is because the high value of the spring stiffness causes a distortion in the lower modes, thereby making several component modes participate significantly in the global modes. However, this distortion becomes smaller in the fourth frequency cluster and negligible in the fifth one. This is explained by noting that the modal coupling becomes small in these bands, in which case the global modes vibrate primarily in the single corresponding component mode. Another interpretation is that the higher modes are stiffer than the lower ones; hence, the coupling stiffness has much less effect on the former than on the latter.

Figure 7 is an interesting illustration of the importance of the constraint location. An assembly of three strongly coupled beams $(\bar{k}=100)$ interconnected at $x_{c}=0.7829$ is considered. The constraint is located at (or very near) the node of the second component mode, i.e., $\phi_{2}\left(x_{c}\right) \simeq 0$. The second band of the tuned assembly has a threefold multiple (or nearly multiple) eigenvalue, as can be seen from Fig. 7 and Eq. (33). This (nearly) multiple eigenvalue is split by mistuning, yielding a narrow cluster. Also note that the last mode of the first group changes position to actually become the sixth mode. This is because the frequencies of the first group are much further apart for $x_{c}=0.78$ than for $x_{c}=1$, and also because the frequencies in the second group are lower for $x_{c}=0.78$ (due to zero coupling in the second group) than for $x_{c}=1$. As expected, because of the large coupling stiffness, the modes of the first group (1st, 2nd, and 6th modes) remain extended. On the other hand, the modes of the second band (3rd, 4th, and 5th modes) become very strongly localized when mistuning is introduced, because the coupling terms in the second rows of the submatrices in Eq. (33) are (nearly) zero, since $\phi_{2}\left(x_{c}\right) \simeq 0$. The modes of the third and fourth groups are only partially localized. Hence, if the beams are interconnected at or near a component mode's node, the corresponding group of global modes become strongly localized when small mistuning is in- troduced, even for very large coupling. Also, some higher groups of modes may not become localized, leading to the concept of "transient" localization (in the modal domain).

\section{Discussion}

The above results show that small disorder may have drastic effects on the dynamics of the system. The degree of localization depends upon the ratio of modal mistuning $\left(\lambda_{i}^{j}\right)$ to modal coupling $\left[R_{i}^{2} \phi_{i}\left(x_{c}\right)\right]$, with localization occurring if both quantities are small and if this ratio is on the order of one or larger. For the beam system, the modal mistuning was considered to be constant, which may or may not be the case for physical systems. The modal coupling is determined by the spring stiffness constant, the component mode number, and the modal deflection at the constraint location. The modal coupling decreases rapidly as the component mode number increases; hence, for arbitrarily large coupling stiffness and arbitrarily small mistuning, strong localization occurs in all modes higher than some threshold number. Also, strong localization occurs in the groups of modes whose primary component mode has a node at or near the constraint location. Localization may or may not occur in the neighboring groups of modes depending on the coupling strength. Hence, localization may appear in a group of modes, then disappear, and eventually reappear in the higher modes.

The criterion formulated in Refs. 5 and 7 for a chain of coupled pendulums can be seen to apply readily to assemblies of component systems, as follows. The modal mistuning directly determines the spread in the natural frequencies of the individual mistuned component systems. Furthermore, the value of the modal coupling $R_{i}^{2} \phi_{i}\left(x_{c}\right)$ determines the width of the frequency bands of the tuned system, as can be seen readily from Eq. (33) and from the natural frequencies listed in Figs. $2-7$. [Note that the band character is lost for large values of $R_{i}^{2} \phi_{i}\left(x_{\mathrm{c}}\right)$.] This is also readily evidenced by the modified perturbation expression of the eigenvalues, Eq. (24). For instance, for small $R_{i}^{2}$ the $i$ th frequency cluster of the tuned system becomes narrow, and for $\phi_{i}\left(x_{c}\right)=0$, multiple eigenvalues occur for the tuned system. Therefore, it can be stated that strong localization occurs in a given group of modes if the width of the corresponding frequency band of the tuned system is on the order of or smaller than the spread in individual frequencies (due to mistuning) of the component systems, and if both quantities are small-a generalization of the criterion originally formulated in Refs. 5 and 7.

Finally, although the above study has shown that strong localization occurs only for small (first-order) values of the modal coupling, it is of interest to investigate what happens in the strong coupling case as the number of component systems is increased. Figure 8 displays selected tuned and mistuned modes of an assembly of beams connected at their tips for $\bar{k}=3$. Mistuning has intentionally been chosen very small, of standard deviation $0.3 \%$. Figure 8 displays the tip displacement of the beams in the first mode of the second frequency cluster for various numbers of beams. For a 30-beam assembly, mistuning has very little effect on the mode shape, as is expected in this strong coupling case. As the number of beams increases to 50 , though, the mode begins to be localized, and for 100 beams the mode shape is localized to a relatively small geometric region of the assembly. It is remarkable that this occurs for a finite modal coupling and a very small mistuning. Further increasing the number of beams would produce more pronounced localization. For larger mistuning of standard deviation $3.0 \%$, localization becomes stronger, as shown in Fig. 9 for assemblies of 30 and 50 beams. Even though localization would only be partial for an assembly of three or four beams, the effect shown in Fig. 9 for 50 beams is, indeed, spectacular.

Such mode localization is referred to as weak localization and was recently studied by Kissel. ${ }^{15}$ Contrary to strong localization that occurs over a few component systems, weak localization requires a large number of substructures to occur and features a slower spatial decay; therefore, it is believed to be 

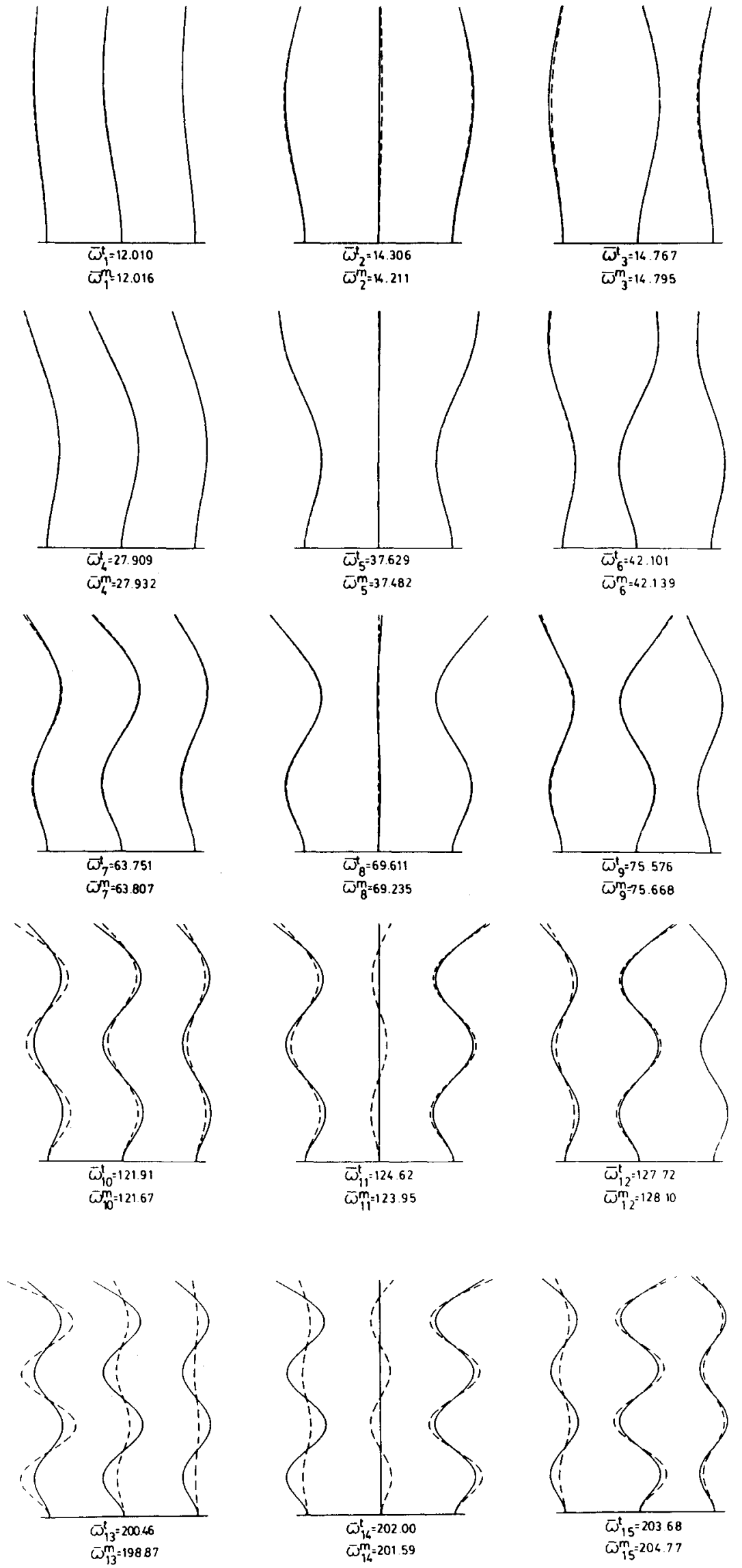

Fig. 6 Modes of tuned $\left(-,, \bar{\omega}^{t}\right)$ and mistuned $\left(---, \bar{\omega}^{m}\right)$ assemblies of three beams for $\bar{k}=100, x_{c}=1$, and $M=7$. Mistuning is as in Fig. 3 . 

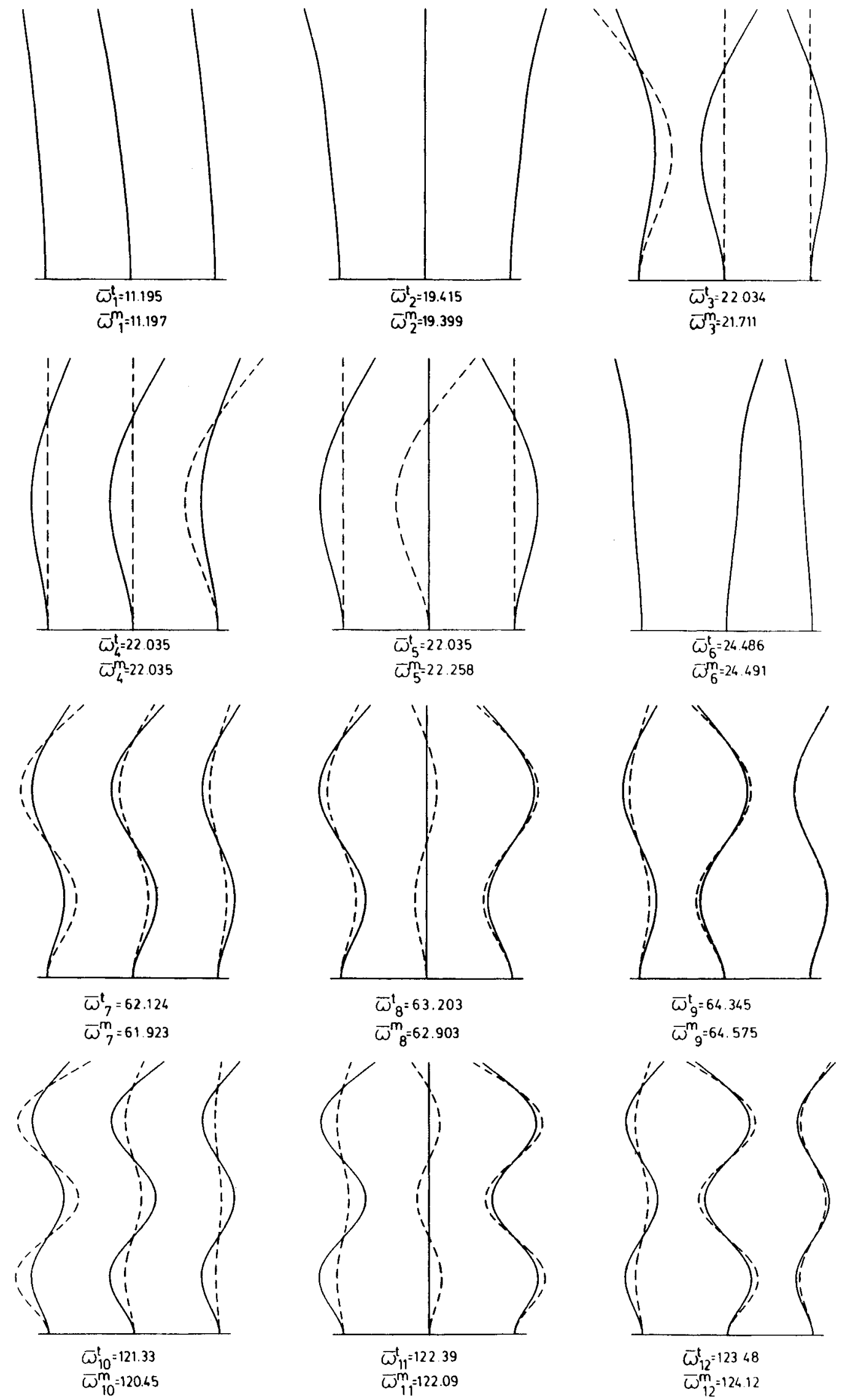

Fig. 7 Modes of tuned $\left(-, \bar{\omega}^{t}\right)$ and mistuned $\left(--,, \bar{\omega}^{m}\right)$ assemblies of three beams for $\bar{k}=100, x_{c}=0.7829$, and $M=7$. Mistuning is as in Fig. 3 . 

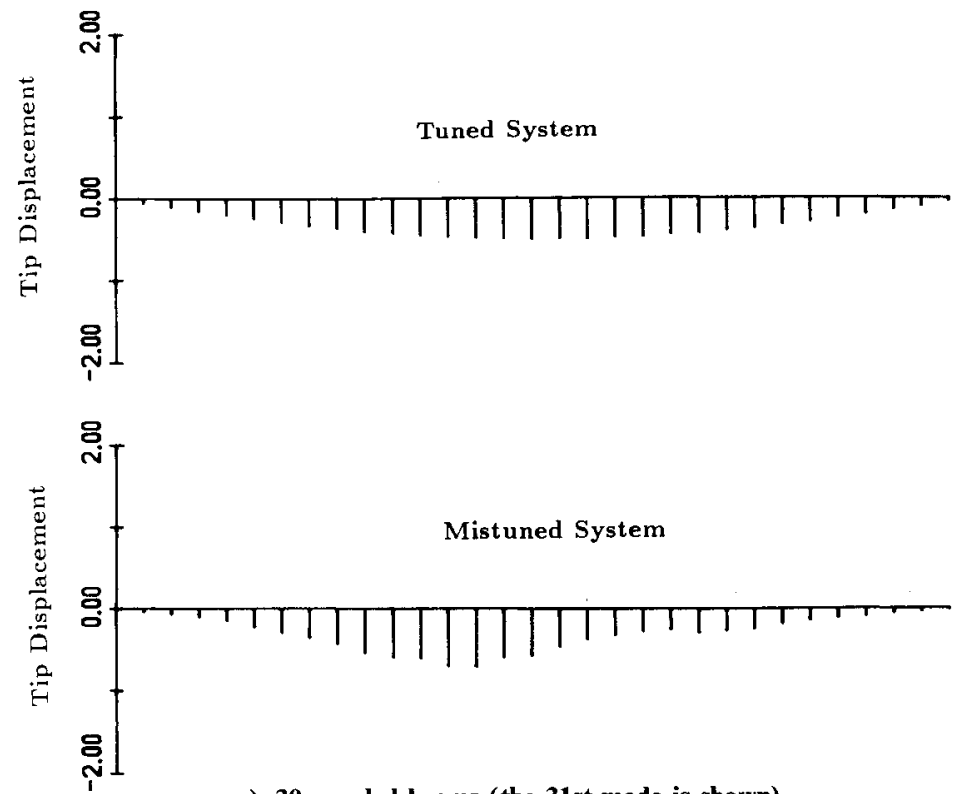

a) 30 coupled beams ( the 31 st mode is shown)
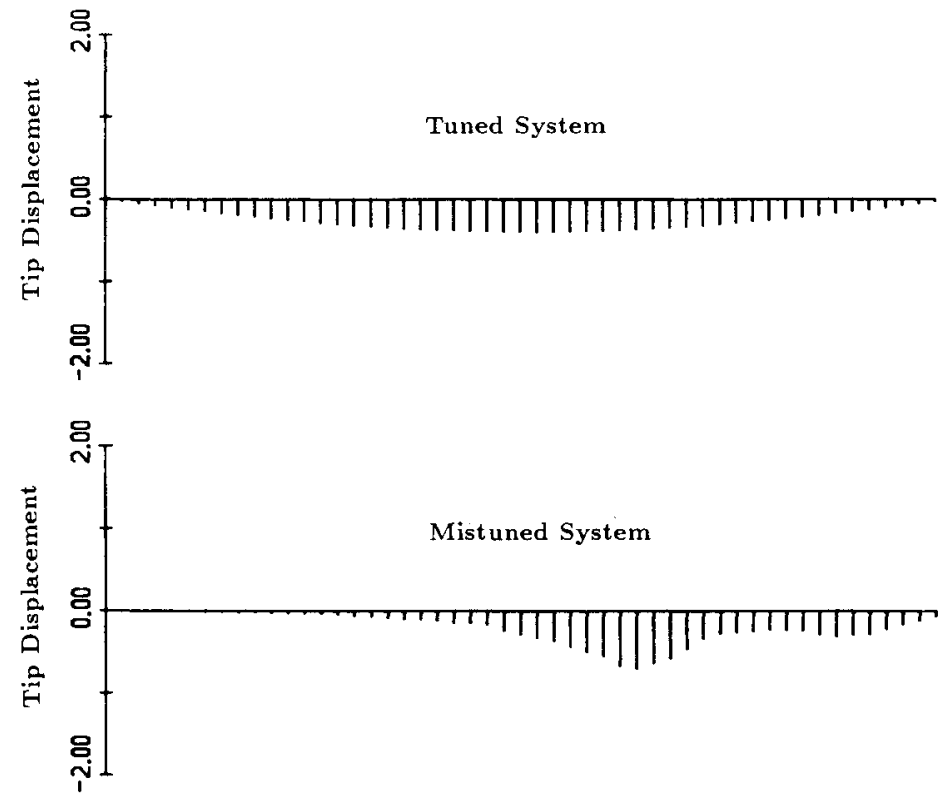

b) 50 beams (51st mode shown)
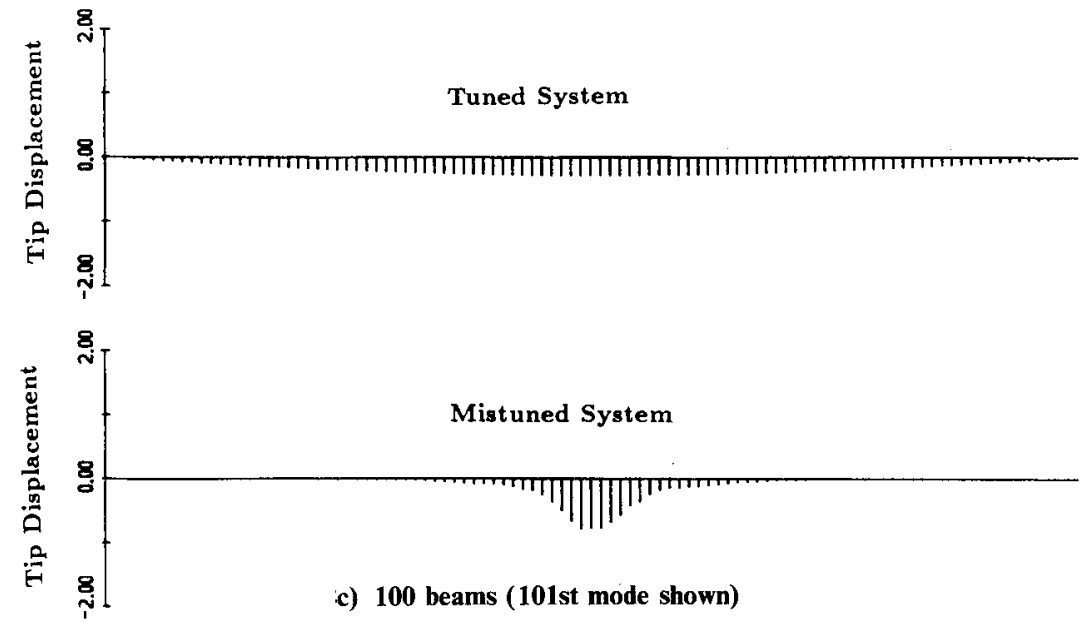

Fig. 8 First mode of the second frequency group of tuned and mistuned assemblies for $\bar{k}=3$ and $x_{c}=1$. The tip displacements of the component beams are shown. The mistuning standard deviation is $0.3 \%$. 

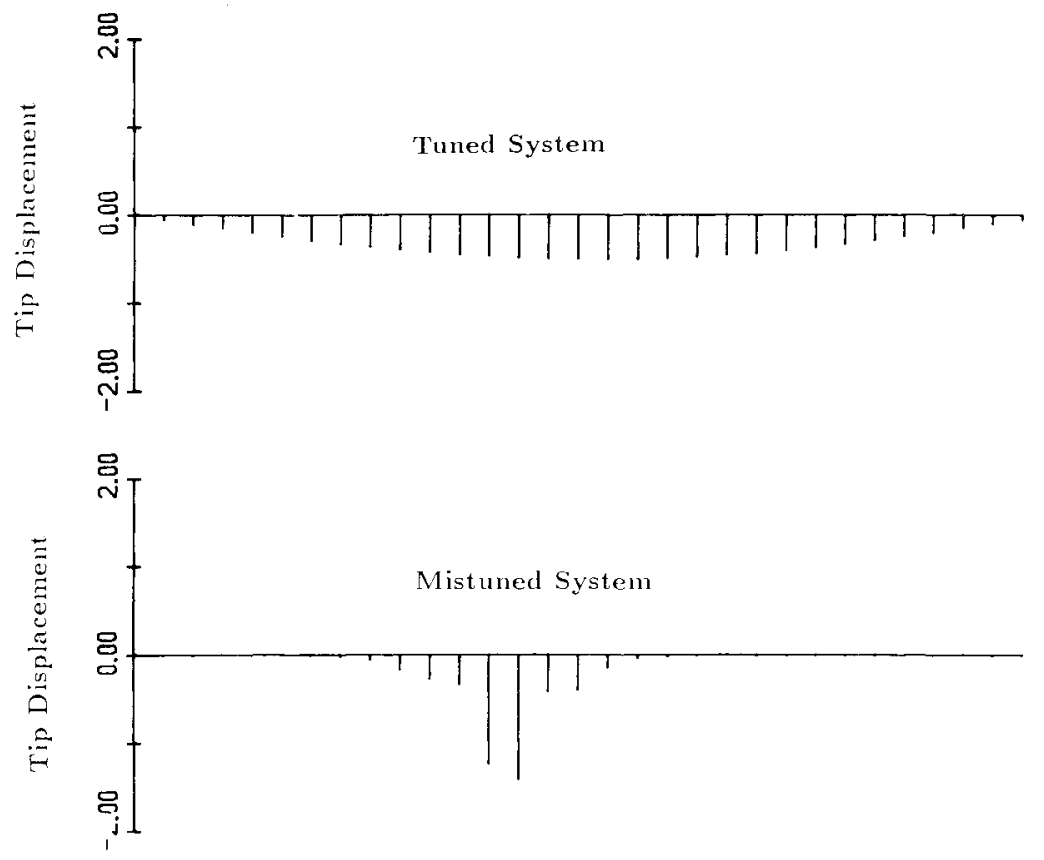

a) 30 beams (31st mode shown)
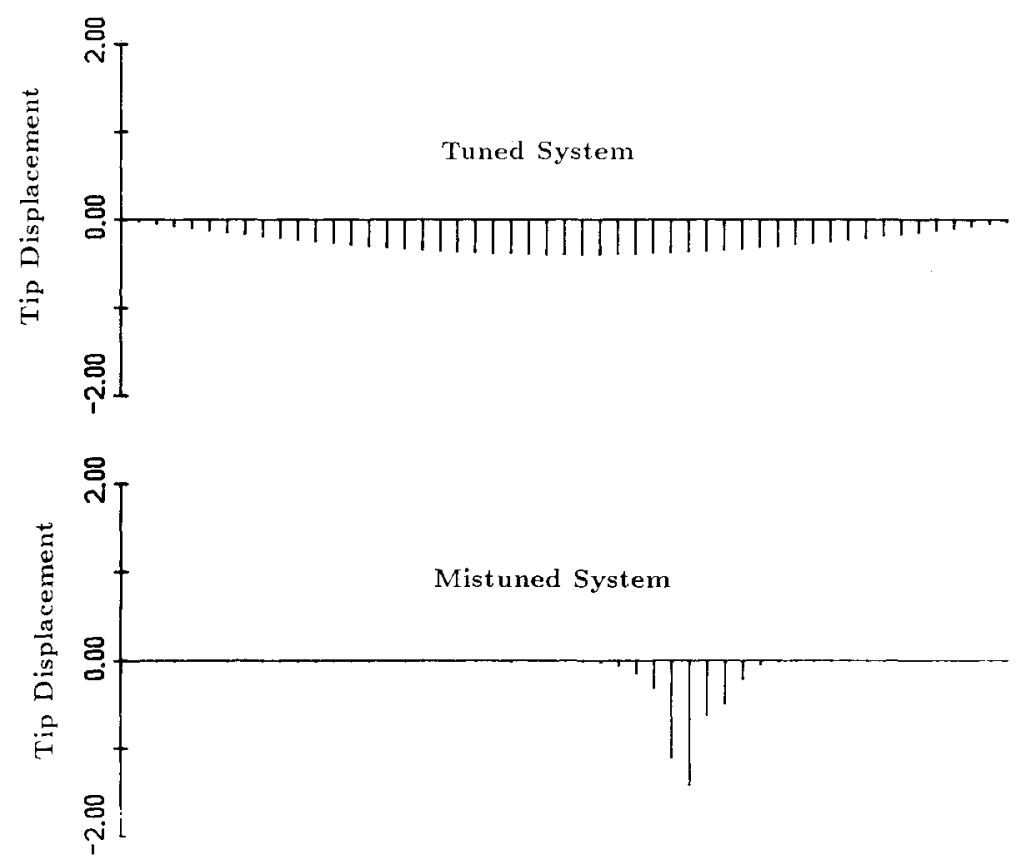

b) $\mathbf{5 0}$ beams (51st mode shown)

Fig. 9 Same as Fig. 8 for a $3 \%$ mistuning standard deviation.

less important than strong localization for most engineering structures, even though weak localization effects could be significant for structures with large numbers of substructures such as bladed-disk assemblies. The occurrence of weak localization also can be tentatively explained by the failure of the classical perturbation analysis. Similarly to strong localization, the perturbation approach fails because the eigenvalues of the tuned system are close, thereby making the corresponding eigenvector perturbation of order one rather than of the first order. The difference is that for strong localization the eigenvalues are close because the modal coupling is small, whereas for weak localization, even though modal coupling is large or finite, the eigenvalues are close because systems with large numbers of substructures have a high modal density. The cumulative effect over $N$ terms produces an eigenvector perturbation of order one rather than of the first order, leading to a localized mode. Increasing the number of substructures, therefore, clearly leads to weak localization, even in the strong coupling case.

\section{Convergence}

The convergence of the component mode analysis has been checked carefully for each of the above calculations. In general, the convergence is excellent, and for the values of the coupling stiffness considered here, the maximum number of component modes necessary to ensure converged frequency values is $M=7$. The component mode analysis converges very quickly when the dimensionless modal couplings $R_{i}^{2} \phi_{i}\left(x_{c}\right)$ are small, since then a single component mode primarily contributes to 
the global modes. The least rapid convergence occurs in the lower modes for large values of $\bar{k}$. Six or seven modes then are necessary to ensure a very good convergence. For example, for $\bar{k}=100$, using seven component modes leads to natural frequencies of the first group of modes converged up to the second decimal place.

\section{Conclusions}

1) Nearly periodic structures such as assemblies of component systems are highly sensitive to small disorder. Under certain conditions, the extended modes of the tuned system become strongly localized when small mistuning is introduced.

2) Combined classical and modified perturbation methods have been developed that predict the occurrence of strong mode localization and analyze the characteristics of localized modes.

3) Strong localization occurs in a given group of modes if the corresponding modal coupling is on the order of or smaller than the modal mistuning. The degree of localization depends only on the ratio of modal coupling to disorder and increases as this ratio decreases.

4) Since the modal coupling decreases as the component mode number increases, localization occurs more easily in the higher groups of modes than in the lower ones. For arbitrarily large coupling stiffness and small mistuning, mode localization is unavoidable if the mode number is large enough. If the coupling constraint is located at the node of a component mode, the corresponding group of modes becomes very strongly localized.

\section{Appendix A: First-Order Eigensolution Perturbation}

The $n$ by $n$ unperturbed real matrix $\left[A_{0}\right]$ has $n$ eigenvalues and right and left eigenvectors denoted by $\lambda_{0 i}, x_{0 i}$, and $y_{0 i}$, respectively, that are assumed to be real. Distinct eigenvalues are assumed. The perturbed matrix $[A]$ is

$$
[A]=\left[A_{0}\right]+[\delta A]+\cdots
$$

where $[\delta A]$ is the first-order perturbation matrix. Considering Taylor expansions of the perturbed eigensolution

$$
\begin{aligned}
& \lambda_{i}=\lambda_{0 i}+\delta \lambda_{i} \\
& \boldsymbol{x}_{i}=\boldsymbol{x}_{0 i}+\delta \boldsymbol{x}_{i}
\end{aligned}
$$

the first-order perturbations $\delta \lambda_{i}$ and $\delta x_{i}$ can be shown to be

$$
\begin{gathered}
\delta \lambda_{i}=\frac{\boldsymbol{y}_{0 i}^{T}[\delta A] \boldsymbol{x}_{0 i}}{\boldsymbol{y}_{0 i}^{T} \boldsymbol{x}_{0 i}}, \quad i=1, \ldots, n \\
\delta \boldsymbol{x}_{i}=\sum_{\substack{j=1 \\
j \neq i}}^{n} \frac{1}{y_{0 j}^{T} \boldsymbol{x}_{0 j}} \frac{y_{0 j}^{T}[\delta A] \boldsymbol{x}_{0 i}}{\lambda_{0 i}-\lambda_{0 j}} x_{0 j}, \quad i=1, \ldots, n
\end{gathered}
$$

Detailed derivations can be found in Refs. 18 and 19.

\section{Appendix B}

$$
\begin{array}{llll}
\sqrt{\bar{\omega}_{1}}=1.875 & \sqrt{\bar{\omega}_{2}}=4.694 & \sqrt{\bar{\omega}_{3}}=7.855 \\
\sqrt{\bar{\omega}_{4}}=10.996 & \sqrt{\bar{\omega}_{5}}=14.137 & \sqrt{\bar{\omega}_{6}}=17.279
\end{array}
$$

\section{Acknowledgment}

This work was supported by National Science Foundation Grant MSM-8700820, Dynamic Systems and Control Program. Dr. Elbert Marsh was the grant monitor. Partial support by a University of Michigan Rackham Faculty Grant and Fellowship is also acknowledged.

\section{References}

'Anderson, P. W., "Absence of Diffusion in Certain Random Lattices," Physical Review, Vol. 109, 1958, pp. 1492-1505.

${ }^{2}$ Rosenstock, H. B. and McGill, R. E., "Vibrational Modes of Disordered Linear Chains," Journal of Mathematical Physics, Vol. 3, 1962, pp. 200-202.

${ }^{3}$ Herbert, D. C. and Jones, R., "Localized States in Disordered Systems," Journal of Physics C: Solid State Physics, Vol. 4, 1971, pp. $1145-1161$.

${ }^{4}$ Ishii, K., "Localization of Eigenstates and Transport Phenomena in the One-Dimensional Disordered System," Supplement of the Progress of Theoretical Physics, Vol. 53, 1973, pp. 77-138.

${ }^{5}$ Hodges, C. H., "Confinement of Vibration by Structural Irregularity," Journal of Sound and Vibration, Vol. 82, No. 3, 1982, pp. 441-424.

'Pierre, C., "Analysis of Structural Systems with Parameter Uncertainties," Ph.D. Dissertation, Duke Univ., Durham, NC, Aug. 1985.

${ }^{7}$ Pierre, C. and Dowell, E. H., "Localization of Vibrations by Structural Irregularity," Journal of Sound and Vibration, Vol. 114, No. 3, May 1987, pp. 549-564.

${ }^{8}$ Valero, N. A. and Bendiksen, O. O." "Vibration Characteristics of Mistuned Shrouded Blade Assemblies," ASME Journal of Engineering for Gas Turbines and Power, Vol. 108, No. 2, 1986, pp. 293-299.

${ }^{9}$ Wei, S. T. and Pierre, C., "Localization Phenomena in Mistuned Assemblies with Cyclic Symmetry. Part I: Free Vibrations," $A S M E$ Journal of Vibration, Acoustics, Stress, and Reliability in Design (to be published).

${ }^{10}$ Bendiksen, O. O., "Mode Localization Phenomena in Large Space Structures," AIAA Journal, Vol. 25, Sept. 1987, pp. 1241-1248.

${ }^{11}$ Cornwell, P. J. and Bendiksen, O. O., "Localization of Vibrations in Large Space Reflectors," AIAA Paper 87-0949, April 1987.

${ }^{12}$ Hodges, C. H. and Woodhouse, J., "Vibration Isolation from Irregularity in a Nearly Periodic Structure: Theory and Measurements," Journal of the Acoustical Society of America, Vol. 74, No. 3, Sept. 1983, pp. 894-905.

${ }^{13}$ Pierre, C., Tang, D. M., and Dowell, E. H., "Localized Vibrations of Disordered Multi-Span Beams: Theory and Experiment," AIAA Journal, Vol. 25, Sept. 1987, pp. 1249-1257.

${ }^{14}$ Pierre, C., "Mode Localization and Eigenvalue Loci Veering Phenomena in Disordered Structures," Journal of Sound and Vibration, Vol. 126, No. 2, Oct. 1988.

${ }^{15}$ Kissel, G. J., "Localization in Disordered Periodic Structures," AIAA Paper 87-0819, April 1987.

${ }^{16}$ Ibrahim, R. A., "Structural Dynamics with Parameter Uncertainties," Applied Mechanics Reviews, Vol. 40, No. 3, March 1987, pp. 309-328.

${ }^{17}$ Dowell, E. H., "Free Vibrations of an Arbitrary Structure in Terms of Component Modes," ASME Journal of Applied Mechanics, Vol. 39, No. 3, Sept. 1972, pp. 727-732.

${ }^{18}$ Meirovitch, L., Computational Methods in Structural Dynamics, Sijthoff \& Noordhoff, The Netherlands, 1980.

${ }^{19}$ Courant, R. and Hilbert, D., Methods of Mathematical Physics, Vol. I, Interscience, New York, 1953.

${ }^{20}$ Pierre, C., "Eigensolution Perturbation for Systems with Perturbed Boundary Conditions," Journal of Sound and Vibration, Vol. 112, Jan. 1987, pp. 167-172. 